\title{
A Review on Real Time Heart Rate Monitoring System using USB camera
}

\author{
Mr. Sachin M. Karmuse, Dr. Arun L. Kakhandki \\ D.K.T.E. Society's Textile \& Engineering Institute, Ichalkaranji, India \\ Vishwanathrao Deshpande Institute Of Technology , Haliyal, India
}

\begin{abstract}
People are becoming more and more interested in their personal health. This is evident from the rapid rise in personal health related applications for smart phones, which today are capable of complex calculations. Vital signs are good indicators of personal health, and measurement of these are of interest. The most commonly measured vital signs are the heart rate and the blood pressure. Research progressing during last decade focuses more on noncontact based systems which are simple, low-cost and comfortable to use. Still most of the noncontact based systems are fit for lab environments in offline situation but needs to progress considerably before they can be applied in real time applications. This paper describe a review of real time method to measure Heart Rate using a USB webcam of a laptop computer. The heart rate is obtained through facial skin color variation caused by blood circulation. Three different signal processing methods such as Fast Fourier Transform (FFT), Independent Component Analysis (ICA) and Principal Component Analysis (PCA) can be applied on the color channels in video recordings. The blood volume pulse (BVP) is extracted from the facial forehead regions. Heart Rate is subsequently quantified and compared to corresponding reference measurements. The obtained results show that there is a high degrees of agreement between the proposed experiments and reference measurements. This technology can be use for advancing personal health care and telemedicine
\end{abstract}

. Ming-Zher Poh, Daniel J. McDuff, and Rosalind W. Picard propose the study of "Advancements in Noncontact, Multiparameter Physiological Measurements Using a Webcam". This is a simple, low-cost method for measuring multiple physiological parameters using a basic webcam. By applying independent component analysis on the color channels in video recordings, extract the blood volume pulse from the facial regions. Heart rate (HR), respiratory rate, and $\mathrm{HR}$ variability (HRV, an index for cardiac autonomic activity) were subsequently quantified and compared to corresponding measurements using Food and Drug administration-approved sensors. This technology is very useful for advancing personal health care and telemedicine.
Hamed Monkaresi, Rafael A. Calvo, and Hong Yan describe "A Machine Learning Approach to improve Contactless Heart Rate Monitoring Using a Webcam" In this paper, he evaluate this technique for measuring $\mathrm{HR}$ in a specific controlled situation, in a natural computer interaction session and in an exercise situation. These reading also compared with HR which was measured simultaneously using an electrocardiography (ECG) device during all sessions. He propose a machine learning approach to improve the accuracy of HR detection in naturalistic measurements. The results demonstrate that the root mean squared error is reduced from 43.76 beats per minute (bpm) to 3.64 (bpm) using his proposed method. The study analyses the problems caused by unwanted movements and the wide dynamic range of HR 
which are common during real world measurement. This paper addressed these issues by building and training specific models for each participant using ML techniques. The results suggest that the kNN based technique outperforms other approaches (manual or computational) that try to select the best independent component for HR estimation. On average the mean of absolute errors in HR estimation is reduced to $0.68 \mathrm{bpm}$ by applying $\mathrm{kNN}$ technique to the ICA outputs among 10 participants in the HCI scenario. The $\mathrm{kNN}$ technique also improved the accuracy of the HR estimation over the former method in indoor exercising conditions.

Alexander M. Chan, Nandakumar Selvaraj, Nima Ferdosi, and Ravi Narasimhan describe "Wireless Patch Sensor for Remote Monitoring of Heart Rate,

Respiration, Activity, and Falls". This paper describe results of a wireless Bluetooth Low Energy (BLE) patch sensor. This sensor consisting of two electrocardiography (ECG) electrodes, a microcontroller, a tri-axial accelerometer, and a BLE transceiver. The sensor measures heart rate, heart rate variability (HRV), respiratory rate, posture, steps, and falls and was evaluated on a total of 25 adult participants who have done breathing exercises, various stretches, walking/running, and simulated falls, stationary cycling. Compared to reference devices, the heart rate measurement had a mean absolute error (MAE) of less than $2 \mathrm{bpm}$, time-domain HRV measurements had an RMS error of less than 15 $\mathrm{ms}$, respiratory rate had an MAE of 1.1 breaths per minute during metronome breathing, posture detection had an accuracy of over $95 \%$ in two of the three patch locations, steps were counted with an absolute error which is less than $5 \%$, and is detected with a sensitivity of $95.2 \%$ and specificity of $100 \%$.

Biju V.G., Anith Mohan, Summayya M Greeshma Rosemary Vilfred, Janita Alice Starina, Ajay Soman describe "A Non Contact Method for Measuring

Heart Rate using a Webcam" . This paper suggest a webcam based, vigorous, inexpensive method of measuring heart rate. Independent component analysis is applied on the RGB colour channels in the video recordings, and then the blood volume pulse is extracted from the facial regions. Heart rate (HR) was then compared with standard measurements such as finger blood volume pulse sensor and it gives high accuracy.

Avinash Parnandi and Ricardo Gutierrez-Osuna describe "Contactless Measurement of Heart Rate Variability from Pupillary Fluctuations". In this paper, he present a proof-of-concept method for measuring one such vital parameter, heart rate variability (HRV), in a contactless fashion from spontaneous fluctuations in papillary diameter. This approach uses a remote eye tracker for imaging and an integro-differential algorithm for segmenting the pupiliris boundary. Then estimate HRV from the relative distribution of energy in the low frequency $(0.04$ to $0.15 \mathrm{~Hz})$ and high frequency $(0.15$ to $0.4 \mathrm{~Hz})$ bands of the power spectrum of the time series of pupillary fluctuations. This method is implemented under a range of breathing conditions and under different illumination levels. Results show a high degree of agreement between our pupillary estimate of HRV and ground truth measurements from an ECGgrade heart rate monitor. These results support the feasibility of estimating HRV in a non-contact, non-invasive fashion.

Christoph Bruser, Kurt Stadlthanner, Stijn de Waele describe "Adaptive Beat-to-Beat Heart Rate Estimation in Ballistocardiograms". $\mathrm{He}$ present a novel algorithm for the detection of individual heart beats and beat-to-beat interval lengths in allistocardiograms (BCGs) from healthy subjects. Unsupervised learning techniques is used to extract the shape of a single heart beat from the BCG. Using this training technique we can learn different parameter and by these learned parameters, the occurrence of individual heart beats in the signal is detected. A final refinement step is used which improves the accuracy of the beat-to-beat interval lengths. This is new approach which can be use for heart rate estimation on a beat to beat basis. The agreement of the proposed algorithm with an ECG reference has been evaluated. A relative beat-to-beat interval error of $1.79 \%$ with a coverage of $95.94 \%$ was achieved on recordings from 16 subjects.

Daniel Lakens describe "Using a Smartphone to Measure Heart Rate Changes during Relived Happiness and Anger". This study describe the 
feasibility of measuring heart rate (HR) differences associated with different emotional states such as happiness and anger with a smart phone. Novice experimenters measured higher HRs during relived anger and happiness (replicating findings in the literature) outside a laboratory environment with a smart phone app that relied on photoplethysmography. This study demonstrates that smart phones can be used to measure HR differences associated with relived experiences of anger and happiness. Smart phones provide an easy-to-use, readily and portable and available measurement tool for emotion researchers. In addition, the current experiment also give experimental result that smart phone applications allow even inexperienced experimenters to collect reliable physiological data. Plethysmographic signals can even be collected from a distance when a camera is positioned 0.5 to $1.5 \mathrm{~m}$ away from an individual. This technique provides new opportunities to apply psychological insights

about the relationship between emotional states in daily life and HR.

Hao-Yu Wu, Michael Rubinstein, Eugene Shih, John Guttag,William Freeman describe "Eulerian Video Magnification for Revealing Subtle Changes in the World". Variations in videos that are impossible to see with the naked eye and display them in an indicative manner. This Paper describe method, which we call Eulerian Video Magnification, takes a standard video sequence as input, and applies spatial decomposition, followed by temporal filtering to the frames. The resulting signal is then amplified to find out hidden information. With this method we can able to visualize the flow of blood as it fills the face and also to amplify and reveal small motions. This is straightforward method that takes a video as input and exaggerates subtle color changes and imperceptible motions. This method does not perform feature tracking or optical flow computation, but merely magnifies temporal color changes using spatio-temporal processing.

Alampreet Chahal describe "A Simple Approach For Heart Rate Monitoring Using Adaptive Filters" (2017). This paper presents a relation between heart rate and vowel speech signal. The proposed method is based on modeling the relationship between speech production of vowel speech signals and heart activities of human. The Adaptive filter is used to manipulate signals to reject unwanted characteristics for accurately monitoring the heart rate. Simulation results show that the proposed method provides more accuracy as compared with existing methods. There is linear relationship between results.

M. K. Islam describe "Study and Analysis of ECG Signal Using MATLAB \& LABVIEW as Effective Tools (2012)". He proposed the study and analysis of ECG signal processing by means of MATLAB tool effectively. Study of ECG signal contain simulation and generation of ECG signal at different states, acquisition of real time ECG data, acquisition of ECG signal filtering \& processing, feature extraction, comparison and analysis between different ECG signal algorithms $\&$ techniques (i.e. Wavlet transform or so). This also cover detection of any abnormalities in ECG, calculating beat rate and so on using the most familiar and multipurpose MATLAB software along with LABVIEW. The proper utilization of MATLAB functions (both built-in and user defined), toolbox and Simulink can lead us to work with ECG signals for processing and analysis both in real time and by simulation with great convenience and accuracy .

Larissa Carvalho describe "Analysis of Heart Rate Monitoring Using a Webcam"(2014).He proposed a non invasive heart rate monitoring system, to monitor subjects of different age groups using Digital Image Processing. This method can be use to visualize the flow of blood as it fills the face. From this result, we can extract the subject's heart rate. The main field of research is Image processing and computer Vision. Variations in videos that are difficult or impossible to see with the naked eye are revealed by taking a standard video of the subject as input, performing face tracking and applying pyramid decomposition, followed by filtering of the frames. The resulting signal is then amplified to find hidden information. We are thus able to visualize the flow of blood as it fills the face. This method is based on the Eulerian Video magnification algorithm presented at SIGGRAPH 2012.

AlladaTirupathi Rao describe "Real Time ECG Signal Transmission for Remote Monitoring" (2017). Main objective of this paper is to implement a monitoring system which monitors 
the heart pulse of a patient. This work presents a novel easy-to-use system intended for the fast and non invasive monitoring of the electrocardiogram (ECG) signal by using a wireless steering wheel. A prototype model is the steering wheel . A heart rate detection algorithm based on mainly on continuous wavelet transform. This wavelet transform has been implemented. This transformation is specially designed to be robust against the most common sources of noise and interference present when acquiring the ECG in the hands. Skin Electrodes were used to record the nerve voltages for detection of heart pulse. The voltages recorded will be sent to an instrumentation amplifier which amplifies the signal, and then to a filter which filters the noise. Thus, analog signal is given to Analog-to-Digital Convertor (ADC) of Arduino. ADC converter convert analog voltages to digital and these digital values can be stored in the EEPROM of Arduino. The values stored in EEPROM will be sent to PC via XBEE (IEEE 802.15.4) wirelessly and a serial port will be opened in the MATLAB by using a serial object. GUI is programmed to make the user interface interactive and simple. Using the real time plot, plotted the values received by XBEE module and making a running waveform which displays when the MATLAB sent a query to Arduino.

Gaurav Kumar describe "Design and Development of Heart Rate and Temperature Measuring Device Using MATLAB" (2017).This paper describes physicians understanding of biosignals as measured with medical instruments becomes the foundation of their decisions and diagnoses of patients, as they rely strongly on what the instruments show. This paper deals with the analysis of PPG (Photo plethysmography) signal to measure heart rate and analysis of resistance change in thermistor for temperature measurement by the means of MATLAB tool effectively. Study of PPG signal include generation of PPG signal, amplification of PPG signal and PPG signal filtering and study of resistance change in thermistor include linearization of the acquired data, measuring the resistance change and interpreting the data. This project is been inspired by the need to find an efficient method for heart rate and temperature analysis which is simple and has good accuracy and less computation time.
H. Rahman describe "Real Time Heart Rate Monitoring From Facial RGB Color Video Using Webcam "(2017). This paper describe a real time HR monitoring method using a USB webcam. The heart rate is obtained through facial skin color variation which is caused by blood circulation. Three different signal processing methods such as Fast Fourier Transform (FFT), Independent Component Analysis (ICA) and Principal Component Analysis (PCA) have been applied on the color channels in video recordings and from that blood volume pulse (BVP) is extracted from region of interest which is facial regions. HR is compared to corresponding reference measurements. The obtained results show that there is a high degrees of agreement between the proposed experiments and reference measurements. This technology can be use for advancing personal health care and telemedicine. Further improvements of the proposed algorithm required which will consider environmental illumination and movement. This improvement can be very useful in many real time applications such as driver monitoring.

N.V. Hoan describe "Real-time Heart Rate Measurement based on Photoplethysmography using Android Smartphone Camera" (2017). This paper describes with the development of smart phone technologies enable photoplethysmogram (PPG) acquisition by camera and heart rate (HR) measurement. This papers describe improved algorithm to extract HR from PPG signal which is recorded by smart phone camera. And also Android application is develop for real-time PPG signal processing. This technique is applied for 400 video samples which is recorded by smart phone camera. These video are imported as input data for further processing and evaluating algorithm on MATLAB. An optimized algorithm is tested on Android platform with different kind of smart phones. Algorithm's performance is assessed by medical device Beurer BC08. According to related works, accuracy parameters includes $90 \%$ number of samples that has relative errors less than 5\%, Person correlation (r) more than 0.9, and standard estimated error ( SEE ) less than 5 beats-per-minutes (bpm).

Conclusion: Biomedical engineering (BME) combines the design and problem-solving skill of engineering with medical and biological sciences 
to improve patient's health care and the quality of life of individuals. Cardiovascular disease is one of the major causes of untimely deaths in the world, heart beat readings are by far the only viable diagnostic tool that could promote early detection of cardiac events. By using this we can measure one's heart rate through fingertip. This paper focuses on the different contact less heart rate monitoring technique Some technique enables us to determine the heart beat rate per minute and then send notification to the mobile phone. It is portable and cost effective. These technique are very easy to handle and also provides great flexibility and serves as a great improvement over other conventional monitoring and alert systems.

\section{References:}

[1] Ming-Zher Poh*, Daniel J. McDuff, and Rosalind W. Picard " Advancements in Noncontact, Multiparameter Physiological Measurements Using a Webcam" IEEE TRANSACTIONS ON BIOMEDICAL ENGINEERING， VOL. 58, NO. 1, JANUARY 2011.

[2] Hamed Monkaresi, Rafael A. Calvo, and Hong Yan "A Machine Learning Approach to Improve Contactless Heart Rate Monitoring Using a Webcam " IEEE Journal of Biomedical and Health Informatics 2013.

[3] Alexander M. Chan, Nandakumar Selvaraj, Nima Ferdosi, and Ravi Narasimhan "Wireless Patch Sensor for Remote Monitoring of Heart Rate, Respiration, Activity, and Falls" 35th Annual International Conference of the IEEE EMBS Osaka, Japan, 3 - 7 July, 2013.

[4] Biju V.G., Anith Mohan, Summayya M Greeshma Rosemary Vilfred, Janita Alice Starina, Ajay Soman " A Non Contact Method for Measuring Heart Rate using a Webcam" International Journal of Engineering Trends and Technology (IJETT) - Volume 34 Number 7- April 2016.

[5] Avinash Parnandi and Ricardo GutierrezOsuna "Contactless Measurement of Heart Rate Variability from Pupillary Fluctuations " Humaine Association
Conference on Affective Computing and Intelligent Interaction 2013

[6] Christoph Br"user, Student Member, IEEE, Kurt Stadlthanner, Stijn de Waele, Member, IEEE, and Steffen Leonhardt, Senior Member, IEEE " Adaptive Beat-toBeat Heart Rate Estimation in Ballistocardiograms " IEEE Tans. Inf. Technol. Biomed., vol. 15, no. 5, pp. 778786, 2011.

[7] Daniel Lakens " Using a Smartphone to Measure Heart Rate Changes during Relived Happiness and Anger" IEEE TRANSACTIONS ON AFFECTIVE COMPUTING, VOL. 4, NO. 2, APRILJUNE 2013.

[8] Hao-Yu Wu, Michael Rubinstein, Eugene Shih, John Guttag, William Freeman "Eulerian Video Magnification for Revealing Subtle Changes in the World " .

[9] Alampreet Chahal1, Silki Baghla2, Gautam Kaushal3, Dr.Rajbir Kaur4, "A SIMPLE APPROACH FOR HEART RATE MONITORING USING ADAPTIVE FILTERS", International Journal of Advance Engineering and Research Development Volume 4, Issue 6, June -2017.

[10] Alampreet Chahal1, Silki Baghla2, Gautam Kaushal3, Dr.Rajbir Kaur4, "A SIMPLE APPROACH FOR HEART RATE MONITORING USING ADAPTIVE FILTERS", International Journal of Advance Engineering and Research Development Volume 4, Issue 6, June -2017.

[11] Gaurav Kumar, Km. ShilpaMaurya, GargiGoyal, "Design and Development of Heart Rate and Temperature Measuring Device Using MATLAB", International Journal of Advanced Research in Electrical, Electronics and Instrumentation Engineering, Vol. 6, Issue 5, May 2017.

[12] H. Rahman, M.U. Ahmed, S. Begum, P. Funk, "Real Time Heart Rate Monitoring From Facial RGB Color Video Using Webcam", The 29th Annual Workshop of the Swedish Artificial Intelligence Society (SAIS). 2-3 June 2016, Malmö, Sweden.

[13] Larissa Carvalho1, Mr. H.G.Virani2, Mr.Shajahan Kutty3, "Analysis of Heart 
Rate Monitoring Using a Webcam", International Journal of Advanced Research in Computer and Communication Engineering Vol.3, May 2014. 